\title{
ON $k$-RAINBOW DOMINATION IN MIDDLE GRAPHS
}

\author{
KIJUNG KIM*®(])
}

\begin{abstract}
Let $G$ be a finite simple graph with vertex set $V(G)$ and edge set $E(G)$. A function $f: V(G) \rightarrow \mathcal{P}(\{1,2, \ldots, k\})$ is a k-rainbow dominating function on $G$ if for each vertex $v \in V(G)$ for which $f(v)=\emptyset$, it holds that $\bigcup_{u \in N(v)} f(u)=\{1,2, \ldots, k\}$. The weight of a $k$-rainbow dominating function is the value $\sum_{v \in V(G)}|f(v)|$. The $k$-rainbow domination number $\gamma_{r k}(G)$ is the minimum weight of a $k$-rainbow dominating function on $G$. In this paper, we initiate the study of $k$-rainbow domination numbers in middle graphs. We define the concept of a middle $k$-rainbow dominating function, obtain some bounds related to it and determine the middle 3-rainbow domination number of some classes of graphs. We also provide upper and lower bounds for the middle 3-rainbow domination number of trees in terms of the matching number. In addition, we determine the 3-rainbow domatic number for the middle graph of paths and cycles.
\end{abstract}

Mathematics Subject Classification. 05C69.

Received February 17, 2021. Accepted October 24, 2021.

\section{INTRODUCTION}

Let $G=(V, E)$ be a connected undirected graph with the vertex set $V=V(G)$ and edge set $E=E(G)$. The order of $G$ is defined as the cardinality of $V$. The open neighborhood of $v \in V(G)$ is the set $N(v)=\{u \in V(G) \mid$ $u v \in E(G)\}$ and the closed neighborhood of $v \in V(G)$ is the set $N[v]:=N(v) \cup\{v\}$. The degree of $v \in V(G)$ is defined as the cardinality of $N(v)$, denoted by $\operatorname{deg}_{G}(v)$. When no confusion arises, we may delete the subscript $G$ in $\operatorname{deg}_{G}(v)$. The maximum degree and minimum degree of $G$ are denoted by $\Delta(G)$ and $\delta(G)$, respectively. We write $P_{n}, C_{n}$ and $K_{n}$ for a path, a cycle and a complete graph, respectively.

In [8], Hamada and Yoshimura defined the middle graph of a graph. The middle graph $M(G)$ of a graph $G$ is the graph obtained by subdividing each edge of $G$ exactly once and joining all these newly introduced vertices of adjacent edges of $G$ (see Fig. 1). The precise definition of $M(G)$ is as follows. The vertex set $V(M(G))$ is $V(G) \cup E(G)$. Two vertices $v, w \in V(M(G))$ are adjacent in $M(G)$ if (i) $v, w \in E(G)$ and $v, w$ are adjacent in $G$ or (ii) $v \in V(G), w \in E(G)$ and $v, w$ are incident in $G$.

In graph domination theory, a set of vertices is selected as guards such that each vertex not selected has a guard as a neighbor. As a generalization of the graph domination, Brešar et al. introduced the concept of $k$-rainbow domination in [3]. In $k$-rainbow domination theory, $k$-different types of guards are required in the neighborhood

Keywords. $k$-rainbow domination number, middle graph, middle $k$-rainbow domination number, matching number, $k$-rainbow domatic number.

Department of Mathematics, Pusan National University, Busan 46241, Republic of Korea.

*Corresponding author: knukkj@pusan.ac.kr 


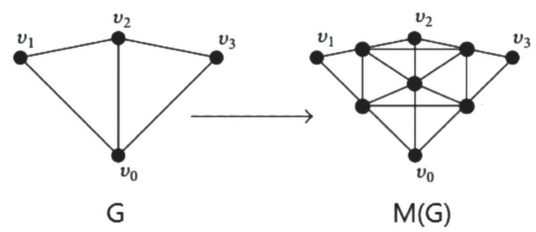

Figure 1. The middle graph $M(G)$ of $G$.

$\{1\}$

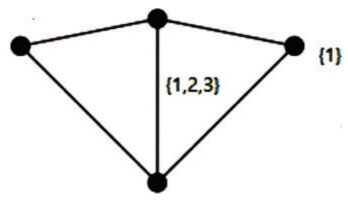

Figure 2. A middle 3-rainbow dominating function on $G$ ( $\emptyset$ is omitted).

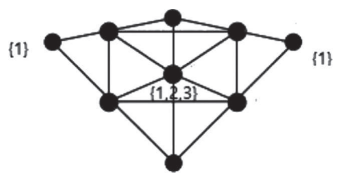

Figure 3. A 3-rainbow dominating function on $M(G)(\emptyset$ is omitted).

of a non-selected vertex. Let $[k]$ be the set of positive integers at most $k$. A function $f: V(G) \rightarrow \mathcal{P}([k])$ is a $k$-rainbow dominating function on $G$ if for each vertex $v \in V(G)$ for which $f(v)=\emptyset$, it holds that $\bigcup_{u \in N(v)} f(u)=[k]$. The weight of a $k$-rainbow dominating function is the value $\sum_{v \in V(G)}|f(v)|$. The $k$-rainbow domination number $\gamma_{r k}(G)$ is the minimum weight of a $k$-rainbow dominating function on $G$. In [4], Chang et al. proved that the $k$-rainbow domination is NP-complete. So, it is worthwhile to determine the $k$-rainbow domination numbers of some classes of graphs. The latest survey on $k$-rainbow domination is introduced in [2]. Indeed, there are many papers on the 2-rainbow domination. For $k \geq 3$, it is more difficult to determine the $k$-rainbow domination number of a graph. The following are a few results on the 3-rainbow domination number. In [10], Shao et al. determined the 3-rainbow domination numbers of paths, cycles and generalized Petersen graphs $P(n, 1)$. In [12], Wang et al. determined the 3-rainbow domination number of $P_{3} \square P_{n}$. In [7], Gao et al. determined the 3-rainbow domination numbers of $C_{3} \square C_{m}$ and $C_{4} \square C_{m}$. In [5], Cynthia et al. determined the 3 -rainbow domination number of circulant graph $G(n ; \pm\{1,2,3\})$. In [6], Furuya et al. proved that for every connected graph $G$ of order $n \geq 8$ with $\delta(G) \geq 2, \gamma_{r 3}(G) \leq \frac{5 n}{6}$.

To study $k$-rainbow domination numbers in the class of middle graphs, we define the following concept. For $v \in V(G)$, we denote $\{e \in E(G) \mid e$ is incident with $v\}$ by $N_{M}(v)$. For $e \in E(G)$, we denote $\{x \in V(G) \cup E(G) \mid x$ is either adjacent or incident with $e\}$ by $N_{M}(e)$. We write $N_{M}[x]=N_{M}(x) \cup\{x\}$. A middle $k$-rainbow dominating function (MkRDF) on a graph $G$ is a function $f: V \cup E \rightarrow \mathcal{P}([k])$ such that every element $x \in V \cup E$ for which $f(x)=\emptyset$ satisfies $\bigcup_{v \in N_{M}(x)} f(v)=[k]$. A middle $k$-rainbow dominating function $f$ gives an ordered partition $\left(V_{0} \cup E_{0}, V_{1} \cup E_{1}, V_{2} \cup E_{2}, \ldots, V_{k} \cup E_{k}\right)$, where $V_{i}:=\{x \in V|| f(x) \mid=i\}$ and $E_{i}:=\{x \in E|| f(x) \mid=i\}$. The weight of a middle $k$-rainbow dominating function $f$ is $\omega(f):=\sum_{x \in V \cup E}|f(x)|$. The middle k-rainbow domination number $\gamma_{r k}^{\star}(G)$ of $G$ is the minimum weight of a middle $k$-rainbow dominating function of $G$. A $\gamma_{r k}^{\star}(G)$-function is a MkRDF on $G$ with weight $\gamma_{r k}^{\star}(G)$. We remark that $\gamma_{r k}^{\star}(G)=\gamma_{r k}(M(G))$ for any graph $G$. As an example, we give Figures 2 and 3. 
In [9], only 2-rainbow domination numbers of the middle graphs were considered. In this paper, we initiate the study of the middle $k$-rainbow domination in graphs. In particular, we determine the exact value of middle 3 -rainbow domination numbers of some classes of graphs. A matching in a graph $G$ is a set of pairwise nonadjacent edges. The maximum number of edges in a matching of a graph $G$ is called the matching number of $G$ and denoted by $\alpha^{\prime}(G)$. We provide upper and lower bounds for the middle 3-rainbow domination number of trees in terms of the matching number. A set $\left\{f_{1}, \ldots, f_{d}\right\}$ of $k$-rainbow dominating functions of $G$ is called a $k$-rainbow dominating family on $G$ if $\sum_{i=1}^{d}\left|f_{i}(v)\right| \leq k$ for each $v \in V(G)$. The maximum number of functions in a $k$-rainbow dominating family on $G$ is the $k$-rainbow domatic number of $G$, denoted by $d_{r k}(G)$. It is known that $k$-rainbow domatic number is well-defined and $d_{r k}(G) \geq k$ for every graph $G$ (see [11]). We determine the 3 -rainbow domatic number for the middle graph of paths and cycles.

In the rest of this section, we present some necessary terminology and notation. For terminology and notation on graph theory not given here, the reader is referred to [1]. Let $T$ be a (rooted) tree. A leaf of $T$ is a vertex of degree one. A pendant edge is an edge incident with a leaf. A support vertex is a vertex adjacent to a leaf. For a vertex $v, C(v)$ denote the set of the children of $v . D[v]$ denote the set of the descendants and $v$. The subtree induced by $D[v]$ is denoted by $T_{v}$. We write $K_{1, n-1}$ for the star of order $n \geq 3$. The double star $\operatorname{DS}_{p, q}$, where $p, q \geq 1$, is the graph obtained by joining the centers of two stars $K_{1, p}$ and $K_{1, q}$. A healthy spider $S_{t, t}$ is the graph from a star $K_{1, t}$ by subdividing each edges of $K_{1, t}$. A wounded spider $S_{t, r}$ is the graph from a star $K_{1, t}$ by subdividing $r$ edges of $K_{1, t}$, where $r \leq t-1$. Note that a star $K_{1, t}$ is a wounded spider $S_{t, 0}$. For a graph $G$ and its subset $S, G-S$ denotes the subgraph of $G$ induced by $V(G) \backslash V(S)$. A diametral path of $G$ is a path with the length which equals the diameter of $G$. The complement of $G=(V, E)$ is the graph $(V, \bar{E})$, which is denoted by $\bar{G}$, where $u v \in \bar{E}$ if and only if $u v \notin E$.

\section{General Bounds of the Middle $k$-RAinbow DOMinAtion Number}

In this section, we obtain general bounds of the middle $k$-rainbow domination number. First, we begin by giving a simple lower bound on the middle $k$-rainbow domination number.

Observation 2.1. If $G$ is a graph with $|V(G)|+|E(G)| \geq k$, then $\gamma_{r k}^{\star}(G) \geq k$.

Proof. Let $f$ be a $\gamma_{r k}^{\star}(G)$-function. If $f(x)=\emptyset$ for some $x \in V(G) \cup E(G)$, then clearly $\bigcup_{y \in N_{M}(x)} f(y)=$ $\{1, \ldots, k\}$. If $f(x) \neq \emptyset$ for all $x \in V(G) \cup E(G)$, then it follows from $|V(G)|+|E(G)| \geq k$ that $\gamma_{r k}^{\star}(G) \geq k$.

Proposition 2.2. Let $G$ be a graph of order $n \geq 2$. Then $\gamma_{r 3}^{\star}(G)=3$ if and only if $G \in\left\{\overline{K_{3}}, P_{2}\right\}$.

Proof. If $G \in\left\{\overline{K_{3}}, P_{2}\right\}$, then clearly $\gamma_{r 3}^{\star}(G)=3$. Conversely, assume that $\gamma_{r 3}^{\star}(G)=3$ and let $f$ be a $\gamma_{r 3}^{\star}(G)$ function. If there exists $x \in V(G) \cup E(G)$ such that $f(x)=[3]$, then $x \in E(G)$ for otherwise $x$ can not dominate the other vertices. Thus, $G=P_{2}$.

Now assume that there is no element with weight 3. If $f(v)=\emptyset$ for some $v \in V(G)$, then there exist at least two edges $e_{1}, e_{2}$ incident to $v$ such that $f\left(e_{1}\right)$ and $f\left(e_{2}\right)$ are not empty. But, end vertices of $e_{1}, e_{2}$ except for $v$ are not dominated, a contradiction. Thus, every vertex in $V(G)$ has non-zero weight so that there are at most three vertices in the graph $G$. One can easily check that $G=\overline{K_{3}}$ or $P_{2}$.

Theorem 2.3. If $G$ is a graph and $v \in V(G)$, then $\gamma_{r k}^{\star}(G)-\min \{\Delta(G)+1, k\} \leq \gamma_{r k}^{\star}(G-v) \leq \gamma_{r k}^{\star}(G)$.

Proof. First, we claim that $\gamma_{r k}^{\star}(G)-\min \{\Delta(G)+1, k\} \leq \gamma_{r k}^{\star}(G-v)$. Let $f$ be a $\gamma_{r k}^{\star}(G-v)$-function. If $k \leq \Delta(G)+1$, then define $g: V(G) \cup E(G) \rightarrow \mathcal{P}([k])$ by $g(v)=[k], g(x)=\emptyset$ for $x \in N_{M}(v)$ and $g(x)=f(x)$ otherwise. If $k>\Delta(G)+1$, then define $g: V(G) \cup E(G) \rightarrow \mathcal{P}([k])$ by $g(x)=\{1\}$ for each $x \in N_{M}[v]$ and $g(x)=f(x)$ otherwise. Clearly, $g$ is a MkRDF of $G$ with weight at most $\gamma_{r k}^{\star}(G-v)+\min \{\Delta(G)+1, k\}$. Thus, $\gamma_{r k}^{\star}(G)-\min \{\Delta(G)+1, k\} \leq \gamma_{r k}^{\star}(G-v)$.

Next, we claim that $\gamma_{r k}^{\star}(G-v) \leq \gamma_{r k}^{\star}(G)$. Let $f$ be a $\gamma_{r k}^{\star}(G)$-function. Define $h: V(G-v) \cup E(G-v) \rightarrow \mathcal{P}([k])$ by $h(u)=f(u) \cup f(u v)$ for $u \in N(v)$ and $h(x)=f(x)$ otherwise. Then clearly $h$ is a MkRDF of $G-v$ with weight $\gamma_{r k}^{\star}(G)$. 
Theorem 2.4. Let $G$ be a graph. Then

(1) $\gamma_{r k}^{\star}(G)-k \leq \gamma_{r k}^{\star}(G+e) \leq \gamma_{r k}^{\star}(G)+1$ for $e \in E(\bar{G})$,

(2) $\gamma_{r k}^{\star}(G)-1 \leq \gamma_{r k}^{\star}(G-e) \leq \gamma_{r k}^{\star}(G)+k$ for $e \in E(G)$.

Proof. (i) First, we show that $\gamma_{r k}^{\star}(G+e) \leq \gamma_{r k}^{\star}(G)+1$. Let $f$ be $\gamma_{r k}^{\star}(G)$-function. Clearly, we can extend $f$ to a MkRDF of $G+e$ by assigning $\{1\}$ to $f(e)$.

Next, we claim that $\gamma_{r k}^{\star}(G)-k \leq \gamma_{r k}^{\star}(G+e)$. Let $f$ be $\gamma_{r k}^{\star}(G+e)$-function and let $e=u v$. If $f(e)=\emptyset$, then clearly the function $\left.f\right|_{V(G) \cup E(G)}$ is a MkRDF of $G$. This implies $\gamma_{r k}^{\star}(G)-k<\gamma_{r k}^{\star}(G) \leq \gamma_{r k}^{\star}(G+e)$. Now assume that $f(e) \neq \emptyset$. Then define $g: V(G) \cup E(G) \rightarrow \mathcal{P}([k])$ by $g(u)=f(u) \cup f(e), g(v)=f(v) \cup f(e)$ and $g(x)=f(x)$ otherwise. Clearly, $g$ ia a MkRDF of $G$ with weight $\gamma_{r k}^{\star}(G+e)+|f(e)|$. Thus, $\gamma_{r k}^{\star}(G)-k \leq$ $\gamma_{r k}^{\star}(G)-|f(e)| \leq \gamma_{r k}^{\star}(G+e)$.

(ii) By (i), $\gamma_{r k}^{\star}(G-e)-k \leq \gamma_{r k}^{\star}((G-e)+e) \leq \gamma_{r k}^{\star}(G-e)+1$. This implies (ii).

Theorem 2.5. Let $T$ be a tree of order $n \geq 3$. Then $\gamma_{r 3}^{\star}(T) \leq \frac{3 n-1}{2}$ with equality if and only if $T \cong P_{3}$.

Proof. We proceed by induction on the order $n$ of $T$. Obviously, the statement is true for a path $P_{3}$.

Let $T$ be a tree of order $n \geq 4$. Suppose that every tree $T^{\prime}$ of order $n^{\prime}(<n)$ satisfies $\gamma_{r 3}^{\star}\left(T^{\prime}\right) \leq \frac{3 n^{\prime}-1}{2}$. Let $f$ be a $\gamma_{r 3}^{\star}\left(T^{\prime}\right)$-function. If $T$ is a star $K_{1, n-1}$, then $\gamma_{r 3}^{\star}(T)=n+1<\frac{3 n-1}{2}$. Assume that $T$ is a double star $\operatorname{DS}_{p, q}$ with $p \geq q \geq 1$. Then $p+q+3=n+1=\gamma_{r 3}^{\star}(T)<\frac{3 n-1}{2}$. Now we assume that $T$ is neither a star or a double star. Then it is easy to see that $T$ has diameter at least four. Among all of diametrical paths in $T$, we choose $x_{0} x_{1}, \ldots, x_{d}$ so that it maximizes the degree of $x_{d-1}$. Root $T$ at $x_{0}$. We divide our consideration into three cases.

Case 1. $\operatorname{deg}_{T}\left(x_{d-1}\right)=t \geq 3$.

Now $T_{x_{d-1}} \cong K_{1, t-1}$. Let $T^{\prime}=T-T_{x_{d-1}}$. Applying the induction hypothesis to $T^{\prime}$, we have $\gamma_{r 3}^{\star}\left(T^{\prime}\right) \leq$ $\frac{3(n-t)-1}{2}$. Define $g: V(T) \cup E(T) \rightarrow \mathcal{P}([3])$ by $g\left(x_{d-1}\right)=g\left(x_{d-2} x_{d-1}\right)=g\left(x_{d}\right)=\emptyset, g\left(x_{d-1} x_{d}\right)=[3]$, $g\left(x_{d-1} x\right)=\emptyset$ and $g(x)=\{1\}$ for $x \in C\left(x_{d-1}\right) \backslash\left\{x_{d}\right\}$ and $g(x)=f(x)$ otherwise. Then clearly $g$ is a M3RDF of $T$ and so

$$
\gamma_{r 3}^{\star}(T) \leq \omega(g)=\gamma_{r 3}^{\star}\left(T^{\prime}\right)+t+1 \leq \frac{3(n-t)-1}{2}+t+1=\frac{3 n-t+1}{2}<\frac{3 n-1}{2} .
$$

Case 2. $\operatorname{deg}_{T}\left(x_{d-1}\right)=2$ and $\operatorname{deg}_{T}\left(x_{d-2}\right)=t \geq 3$.

Now $T_{x_{d-2}} \cong S_{t-1, r}$, where $1 \leq r \leq t-1$. Let $T^{\prime}=T-T_{x_{d-2}}$. Applying the induction hypothesis to $T^{\prime}$, we have $\gamma_{r 3}^{\star}\left(T^{\prime}\right) \leq \frac{3(n-t-r)-1}{2}$. Define $g: V(T) \cup E(T) \rightarrow \mathcal{P}([3])$ by $g\left(x_{d-3} x_{d-2}\right)=g\left(x_{d-2}\right)=g\left(x_{d-1}\right)=$ $g\left(x_{d-1} x_{d}\right)=\emptyset, g\left(x_{d-2} x_{d-1}\right)=[3], g\left(x_{d}\right)=\{1\}, g\left(x_{d-2} x\right)=\emptyset$ for $x \in C\left(x_{d-2}\right) \backslash\left\{x_{d-1}\right\}, g(x)=\{1\}$ if $x \in C\left(x_{d-2}\right) \backslash\left\{x_{d-1}\right\}$ is a leaf, $g(x)=\emptyset, g(x y)=[3]$ and $g(y)=\emptyset$ if $x \in C\left(x_{d-2}\right) \backslash\left\{x_{d-1}\right\}$ is a support vertex, where $y \in C(x)$, and $g(x)=f(x)$ otherwise. Then clearly $g$ is a M3RDF of $T$ and so

$$
\gamma_{r 3}^{\star}(T) \leq \omega(g)=\gamma_{r 3}^{\star}\left(T^{\prime}\right)+t+2 r \leq \frac{3(n-t-r)-1}{2}+t+2 r=\frac{3 n-t+r-1}{2}<\frac{3 n-1}{2} .
$$

Case 3. $\operatorname{deg}_{T}\left(x_{d-1}\right)=2$ and $\operatorname{deg}_{T}\left(x_{d-2}\right)=2$.

Now $T_{x_{d-2}} \cong P_{3}$. Let $T^{\prime}=T-T_{x_{d-2}}$. Applying the induction hypothesis to $T^{\prime}$, we have $\gamma_{r 3}^{\star}\left(T^{\prime}\right) \leq \frac{3(n-3)-1}{2}$. Define $g: V(T) \cup E(T) \rightarrow \mathcal{P}([3])$ by $g\left(x_{d-3} x_{d-2}\right)=g\left(x_{d-2}\right)=g\left(x_{d-1}\right)=g\left(x_{d-1} x_{d}\right)=\emptyset, g\left(x_{d-2} x_{d-1}\right)=[3]$, $g\left(x_{d}\right)=\{1\}$ and $g(x)=f(x)$ otherwise. Then clearly $g$ is a M3RDF of $T$ and so

$$
\gamma_{r 3}^{\star}(T) \leq \omega(g)=\gamma_{r 3}^{\star}\left(T^{\prime}\right)+4 \leq \frac{3(n-3)-1}{2}+4=\frac{3 n-2}{2}<\frac{3 n-1}{2} .
$$




\section{The middle 3-Rainbow domination number of PAThs, CyCles And COMPlete GRAPHS}

In this section, we determine the middle 3-rainbow domination number of paths, cycles and complete graphs.

Proposition 3.1. For $n \geq 2$,

$$
\gamma_{r 3}^{\star}\left(P_{n}\right)= \begin{cases}\frac{4 n-1}{3} & \text { for } n \equiv 1(\bmod 3) ; \\ \frac{4 n+1}{3} & \text { for } n \equiv 2(\bmod 3) ; \\ \frac{4 n}{3} & \text { for } n \equiv 0(\bmod 3) .\end{cases}
$$

Proof. One can check that $\gamma_{r 3}^{\star}\left(P_{2}\right)=3, \gamma_{r 3}^{\star}\left(P_{3}\right)=4, \gamma_{r 3}^{\star}\left(P_{4}\right)=5, \gamma_{r 3}^{\star}\left(P_{5}\right)=7$ and $\gamma_{r 3}^{\star}\left(P_{6}\right)=8$. We proceed by induction on $n$. Assume that $P_{n}=v_{1} v_{2}, \ldots, v_{n}$ and let $x_{2 i-1}=v_{i}$ for $1 \leq i \leq n$ and $x_{2 i}=v_{i} v_{i+1}$ for $1 \leq i \leq n-1$. Let $V\left(P_{n}\right) \cup E\left(P_{n}\right)=\left\{x_{1}, \ldots, x_{2 n-1}\right\}$ and $f$ be a $\gamma_{r 3}^{\star}\left(P_{n}\right)$-function. We divide our consideration into three cases.

Case 1. $n \equiv 1(\bmod 3)$.

First, we claim that $\gamma_{r 3}^{\star}\left(P_{n}\right) \geq \frac{4 n-1}{3}$. Assume that $n \geq 7$. It is easy to see that $\sum_{i=1}^{5}\left|f\left(x_{i}\right)\right| \geq 3$. If $\sum_{i=1}^{5}\left|f\left(x_{i}\right)\right|=3$ and $\left|f\left(x_{4}\right)\right|+\left|f\left(x_{5}\right)\right|=0$, then, to dominate $x_{5}$, it must be $f\left(x_{6}\right)=\{1,2,3\}$. Thus, it is easy to see that $\sum_{i=1}^{7}\left|f\left(x_{i}\right)\right| \geq 6$. Define $h: V\left(P_{n}-\left\{v_{1}, \ldots, v_{4}\right\}\right) \cup E\left(P_{n}-\left\{v_{1}, \ldots, v_{4}\right\}\right) \rightarrow \mathcal{P}([3])$ by $h\left(x_{9}\right)=f\left(x_{8}\right) \cup f\left(x_{9}\right)$ and $h\left(x_{i}\right)=f\left(x_{i}\right)$ for $10 \leq i \leq 2 n-1$. Clearly, $h$ is a M3KDF of $P_{n-4}$ with weight at most $\omega(f)-6$. By the induction hypothesis, we have

$$
\gamma_{r 3}^{\star}\left(P_{n}\right) \geq \omega(h)+6 \geq \gamma_{r 3}^{\star}\left(P_{n-4}\right)+6=\frac{4(n-4)}{3}+6=\frac{4 n+2}{3} .
$$

If $\sum_{i=1}^{5}\left|f\left(x_{i}\right)\right|=3$ and $\left|f\left(x_{4}\right)\right|+\left|f\left(x_{5}\right)\right| \neq 0$, then there is one possibility, namely $\left|f\left(x_{1}\right)\right|=1,\left|f\left(x_{2}\right)\right|=0$, $\left|f\left(x_{3}\right)\right|=1,\left|f\left(x_{4}\right)\right|=1$ and $\left|f\left(x_{5}\right)\right|=0$. To dominate $x_{5}$, it must be $\left|f\left(x_{6}\right)\right|=2$. Thus, it is easy to see that $\sum_{i=1}^{7}\left|f\left(x_{i}\right)\right| \geq 5$. Define $h: V\left(P_{n}-\left\{v_{1}, \ldots, v_{4}\right\}\right) \cup E\left(P_{n}-\left\{v_{1}, \ldots, v_{4}\right\}\right) \rightarrow \mathcal{P}([3])$ by $h\left(x_{9}\right)=f\left(x_{8}\right) \cup f\left(x_{9}\right)$ and $h\left(x_{i}\right)=f\left(x_{i}\right)$ for $10 \leq i \leq 2 n-1$. Clearly, $h$ is a M3KDF of $P_{n-4}$ with weight at most $\omega(f)-5$. By the induction hypothesis, we have

$$
\gamma_{r 3}^{\star}\left(P_{n}\right) \geq \omega(h)+6 \geq \gamma_{r 3}^{\star}\left(P_{n-4}\right)+5=\frac{4(n-4)}{3}+5=\frac{4 n-1}{3} .
$$

Now assume that $\sum_{i=1}^{5}\left|f\left(x_{i}\right)\right| \geq 4$. Define $g: V\left(P_{n}-\left\{v_{1}, v_{2}, v_{3}\right\}\right) \cup E\left(P_{n}-\left\{v_{1}, v_{2}, v_{3}\right\}\right) \rightarrow \mathcal{P}([3])$ by $g\left(x_{7}\right)=f\left(x_{6}\right) \cup f\left(x_{7}\right)$ and $g\left(x_{i}\right)=f\left(x_{i}\right)$ for $8 \leq i \leq 2 n-1$. Clearly, $g$ is a M3KDF of $P_{n-3}$ with weight at most $\omega(f)-4$. By the induction hypothesis, we have

$$
\gamma_{r 3}^{\star}\left(P_{n}\right) \geq \omega(g)+4 \geq \gamma_{r 3}^{\star}\left(P_{n-3}\right)+4=\frac{4(n-3)-1}{3}+4=\frac{4 n-1}{3} .
$$

Next, we claim that $\gamma_{r 3}^{\star}\left(P_{n}\right) \leq \frac{4 n-1}{3}$. Define $h: V\left(P_{n}\right) \cup E\left(P_{n}\right) \rightarrow \mathcal{P}([3])$ by $h\left(v_{1+3 i}\right)=\{1\}$ for $0 \leq i \leq \frac{n-1}{3}$, $h\left(x_{4+6 i}\right)=[3]$ for $0 \leq i \leq \frac{n-4}{3}$ and $h(x)=\emptyset$ otherwise. It is easy to see that $h$ is a M3RDF of $P_{n}$ with weight $\frac{4 n-1}{3}$. Thus, we have $\gamma_{r 3}^{\star}\left(P_{n}\right)=\frac{4 n-1}{3}$.

Case 2. $n \equiv 2(\bmod 3)$.

By the same argument as in Case 1, we can show that $\gamma_{r 3}^{\star}\left(P_{n}\right) \geq \frac{4 n+1}{3}$. Define $g: V\left(P_{n}\right) \cup E\left(P_{n}\right) \rightarrow \mathcal{P}([3])$ by $g\left(v_{1+3 i}\right)=\{1\}$ for $0 \leq i \leq \frac{n-2}{3}, g\left(x_{4+6 i}\right)=[3]$ for $0 \leq i \leq \frac{n-5}{3}, g\left(v_{n}\right)=\{2,3\}$ and $g(x)=\emptyset$ otherwise. It is easy to see that $g$ is a M3RDF of $P_{n}$ with weight $\frac{4 n+1}{3}$. Thus, we have $\gamma_{r 3}^{\star}\left(P_{n}\right)=\frac{4 n+1}{3}$.

Case 3. $n \equiv 0(\bmod 3)$.

By the same argument as in Case 1 , we can show that $\gamma_{r 3}^{\star}\left(P_{n}\right) \geq \frac{4 n}{3}$. Define $h: V\left(P_{n}\right) \cup E\left(P_{n}\right) \rightarrow \mathcal{P}([3])$ by $h\left(v_{1+3 i}\right)=\{1\}$ for $0 \leq i \leq \frac{n-3}{3}, h\left(x_{4+6 i}\right)=[3]$ for $0 \leq i \leq \frac{n-3}{3}$ and $h(x)=\emptyset$ otherwise. It is easy to see that $h$ is a M3RDF of $P_{n}$ with weight $\frac{4 n}{3}$. Thus, we have $\gamma_{r 3}^{\star}\left(P_{n}\right)=\frac{4 n}{3}$. 
Proposition 3.2. For $n \geq 3$,

$$
\gamma_{r 3}^{\star}\left(C_{n}\right)= \begin{cases}\frac{4 n+2}{3} & \text { for } n \equiv 1(\bmod 3) ; \\ \frac{4 n^{3}}{3} & \text { for } n \equiv 2(\bmod 3) ; \\ \frac{4 n^{3}}{3} & \text { for } n \equiv 0(\bmod 3) .\end{cases}
$$

Proof. Assume that $C_{n}=v_{1} v_{2}, \ldots, v_{n} v_{1}$, where the subscript $k$ of $v_{k}$ is read by modulo $n$. Let $f$ be a $\gamma_{r 3}^{\star}\left(C_{n}\right)$ function such that the size of $N:=\left\{v_{i} \mid f\left(v_{i}\right) \neq \emptyset\right\}$ is as small as possible. We divide our consideration into three cases.

Case 1. $n \equiv 1(\bmod 3)$.

If there exists some $k$ such that $\left|f\left(v_{k}\right)\right|+\left|f\left(v_{k} v_{k+1}\right)\right|+\left|f\left(v_{k+1}\right)\right| \geq 3$, then define $g: V\left(C_{n}-\left\{v_{k}, v_{k+1}\right\}\right) \cup$ $E\left(C_{n}-\left\{v_{k}, v_{k+1}\right\}\right) \rightarrow \mathcal{P}([3])$ by $g\left(v_{k-1}\right)=f\left(v_{k-1}\right) \cup f\left(v_{k-1} v_{k}\right), g\left(v_{k+2}\right)=f\left(v_{k+1} v_{k+2}\right) \cup f\left(v_{k+2}\right)$ and $g(x)=f(x)$ otherwise. It is easy to see that $g$ is a M3RDF of $P_{n-2}$ with weight at most $\gamma_{r 3}^{\star}\left(C_{n}\right)-3$. By Proposition 3.1, we have

$$
\gamma_{r 3}^{\star}\left(C_{n}\right) \geq \omega(g)+3 \geq \gamma_{r 3}^{\star}\left(P_{n-2}\right)+3=\frac{4(n-2)+1}{3}+3=\frac{4 n+2}{3} .
$$

Assume that

$$
\left|f\left(v_{k}\right)\right|+\left|f\left(v_{k} v_{k+1}\right)\right|+\left|f\left(v_{k+1}\right)\right| \leq 2
$$

for each $k \in[n]$.

If there exists some $k \in[n]$ such that $\left|f\left(v_{k}\right)\right| \geq 2$, then define $g: V\left(C_{n}-v_{k}\right) \cup E\left(C_{n}-v_{k}\right) \rightarrow \mathcal{P}([3])$ by $g\left(v_{k-1}\right)=f\left(v_{k-1}\right) \cup f\left(v_{k-1} v_{k}\right), g\left(v_{k+1}\right)=f\left(v_{k+1}\right) \cup f\left(v_{k} v_{k+1}\right)$ and $g(x)=f(x)$ otherwise. Clearly, $g$ is a M3RDF of $P_{n-1}$ with weight at most $\gamma_{r 3}^{\star}\left(C_{n}\right)-2$. By Proposition 3.1, we have

$$
\gamma_{r 3}^{\star}\left(C_{n}\right) \geq \omega(g)+2 \geq \gamma_{r 3}^{\star}\left(P_{n-1}\right)+2 \geq \frac{4(n-1)}{3}+2=\frac{4 n+2}{3} .
$$

Assume that $\left|f\left(v_{k}\right)\right| \leq 1$ for each $k \in[n]$. If $\left|f\left(v_{k}\right)\right|=0$ for each $k \in[n]$, then to dominate $v_{k}$, we must have $f\left(v_{k-1} v_{k}\right) \cup f\left(v_{k} v_{k+1}\right)=[3]$. Thus, it follows from $n \geq 4$ that

$$
\gamma_{r 3}^{\star}\left(C_{n}\right)=\frac{1}{2} \sum_{1 \leq k \leq n} \sum_{x \in N_{M}\left(v_{k}\right)}|f(x)|=\frac{3 n}{2} \geq \frac{4 n+2}{3} .
$$

Now assume that $N$ is not empty. For a fixed $v_{i} \in N$, if $\left|f\left(v_{i}\right)\right|+\left|f\left(v_{i} v_{i+1}\right)\right|+\left|f\left(v_{i+1}\right)\right|=1$, then $v_{i+1} v_{i+2}$ must dominate $v_{i+1}$ so that $f\left(v_{i+1} v_{i+2}\right)=[3]$, a contradiction to (3.1). Thus, $\left|f\left(v_{i}\right)\right|+\left|f\left(v_{i} v_{i+1}\right)\right|+\left|f\left(v_{i+1}\right)\right|=$ 2. By the same argument, we have $\left|f\left(v_{i}\right)\right|+\left|f\left(v_{i-1} v_{i}\right)\right|+\left|f\left(v_{i-1}\right)\right|=2$. Suppose that $f\left(v_{i-1}\right) \neq \emptyset$ and $f\left(v_{i+1}\right) \neq \emptyset$. Without loss of generality, assume that $f\left(v_{i}\right)=\{1\}, f\left(v_{i-1}\right)=\{3\}$ and $f\left(v_{i+1}\right)=\{2\}$. Then to dominate $v_{i-1} v_{i}$ and $v_{i} v_{i+1}$, we must have $f\left(v_{i-2} v_{i-1}\right)=\{2\}$ and $f\left(v_{i+1} v_{i+2}\right)=\{3\}$. It follows from (3.1) that $f\left(v_{i-2}\right)=f\left(v_{i+2}\right)=\emptyset$. To dominate $v_{i-2}$ and $v_{i+2}$, we must have $f\left(v_{i-3} v_{i-2}\right)=\{1,3\}$ and $f\left(v_{i+2} v_{i+3}\right)=\{1,2\}$. It follows from (3.1) that $f\left(v_{i-3}\right)=f\left(v_{i+3}\right)=\emptyset$. To dominate $v_{i-3}$ and $v_{i+3}$, we must have $\{2\} \subseteq f\left(v_{i-4} v_{i-3}\right)$ and $\{3\} \subseteq f\left(v_{i+3} v_{i+4}\right)$. Define $g: V\left(C_{n}\right) \cup E\left(C_{n}\right) \rightarrow \mathcal{P}([3])$ by $g\left(v_{i-3}\right)=g\left(v_{i+3}\right)=$ $\{1\}, g\left(v_{i-2} v_{i-1}\right)=g\left(v_{i+1} v_{i+2}\right)=[3], g\left(v_{i-3} v_{i-2}\right)=g\left(v_{i-2}\right)=g\left(v_{i-1}\right)=g\left(v_{i-1} v_{i}\right)=g\left(v_{i} v_{i+1}\right)=g\left(v_{i+1}\right)=$ $g\left(v_{i+2}\right)=g\left(v_{i+2} v_{i+3}\right)=\emptyset$ and $g(x)=f(x)$ otherwise. Then $g$ is a M3RDF of $C_{n}$ with weight $\omega(f)$. As above, this implies that $\gamma_{r 3}^{\star}\left(C_{n}\right) \geq \frac{4 n+2}{3}$.

Now assume that for each $v_{i} \in N,\left|f\left(v_{i-1} v_{i}\right)\right|=1$ or $\left|f\left(v_{i} v_{i+1}\right)\right|=1$. Since $\sum_{x \in N_{M}\left[v_{i}\right]}|f(x)| \geq 2$ for $v_{i} \in N$ and $\sum_{x \in N_{M}\left[v_{i}\right]}|f(x)|=3$ for $v_{i} \in V\left(C_{n}\right) \backslash N$, we have

$$
\gamma_{r 3}^{\star}\left(C_{n}\right) \geq \frac{3(n-t)}{2}+\left(t+\frac{t}{2}\right)=\frac{3 n}{2} \geq \frac{4 n+2}{3},
$$

where $t=|N|$.

By Theorem 2.4, $\gamma_{r 3}^{\star}\left(C_{n}\right) \leq \gamma_{r 3}^{\star}\left(P_{n}\right)+1$. Thus, we have $\gamma_{r 3}^{\star}\left(C_{n}\right)=\frac{4 n+2}{3}$. 
Case 2. $n \equiv 2(\bmod 3)$.

If there exists some $k$ such that $\left|f\left(v_{k}\right)\right|+\left|f\left(v_{k} v_{k+1}\right)\right|+\left|f\left(v_{k+1}\right)\right| \geq 3$, then define $g: V\left(C_{n}-\left\{v_{k}, v_{k+1}\right\}\right) \cup$ $E\left(C_{n}-\left\{v_{k}, v_{k+1}\right\}\right) \rightarrow \mathcal{P}([3])$ by $g\left(v_{k-1}\right)=f\left(v_{k-1}\right) \cup f\left(v_{k-1} v_{k}\right), g\left(v_{k+2}\right)=f\left(v_{k+1} v_{k+2}\right) \cup f\left(v_{k+2}\right)$ and $g(x)=f(x)$ otherwise. It is easy to see that $g$ is a M3RDF of $P_{n-2}$ with weight at most $\gamma_{r 3}^{\star}\left(C_{n}\right)-3$. By Proposition 3.1, we have

$$
\gamma_{r 3}^{\star}\left(C_{n}\right) \geq \omega(g)+3 \geq \gamma_{r 3}^{\star}\left(P_{n-2}\right)+3=\frac{4(n-2)}{3}+3=\frac{4 n+1}{3} .
$$

Now suppose that

$$
\left|f\left(v_{k}\right)\right|+\left|f\left(v_{k} v_{k+1}\right)\right|+\left|f\left(v_{k+1}\right)\right| \leq 2
$$

for each $k \in[n]$.

If there exists some $k \in[n]$ such that $\left|f\left(v_{k}\right)\right| \geq 2$, then define $g: V\left(C_{n}-v_{k}\right) \cup E\left(C_{n}-v_{k}\right) \rightarrow \mathcal{P}([3])$ by $g\left(v_{k-1}\right)=f\left(v_{k-1}\right) \cup f\left(v_{k-1} v_{k}\right), g\left(v_{k+1}\right)=f\left(v_{k+1}\right) \cup f\left(v_{k} v_{k+1}\right)$ and $g(x)=f(x)$ otherwise. Clearly, $g$ is a M3RDF of $P_{n-1}$ with weight at most $\gamma_{r 3}^{\star}\left(C_{n}\right)-2$. By Proposition 3.1, we have

$$
\gamma_{r 3}^{\star}\left(C_{n}\right) \geq \omega(g)+2 \geq \gamma_{r 3}^{\star}\left(P_{n-1}\right)+2 \geq \frac{4(n-1)-1}{3}+2=\frac{4 n+1}{3} .
$$

Assume that $\left|f\left(v_{k}\right)\right| \leq 1$ for each $k \in[n]$. By the same argument as Case 1 , we have $\gamma_{r 3}^{\star}\left(C_{n}\right) \geq \frac{4 n+1}{3}$.

Define $h: V\left(C_{n}\right) \cup E\left(C_{n}\right) \rightarrow \mathcal{P}([3])$ by $h\left(v_{1+3 i}\right)=\{1\}$ for $0 \leq i \leq \frac{n-6}{3}, h\left(v_{2+3 i} v_{3+3 i}\right)=[3]$ for $0 \leq i \leq \frac{n-6}{3}$, $h\left(v_{n-1} v_{n}\right)=[3]$ and $h(x)=\emptyset$ otherwise. It is easy to see that $h$ is a M3RDF of $C_{n}$ with weight $\frac{4 n+1}{3}$. Thus, we have $\gamma_{r 3}^{\star}\left(C_{n}\right)=\frac{4 n+1}{3}$.

Case 3. $n \equiv 0(\bmod 3)$.

If there exists some $k \in[n]$ such that $\left|f\left(v_{k}\right)\right| \geq 1$, then define $g: V\left(C_{n}-v_{k}\right) \cup E\left(C_{n}-v_{k}\right) \rightarrow \mathcal{P}([3])$ by $g\left(v_{k-1}\right)=f\left(v_{k-1}\right) \cup f\left(v_{k-1} v_{k}\right), g\left(v_{k+1}\right)=f\left(v_{k+1}\right) \cup f\left(v_{k} v_{k+1}\right)$ and $g(x)=f(x)$ otherwise. Clearly, $g$ is a M3RDF of $P_{n-1}$ with weight at most $\gamma_{r 3}^{\star}\left(C_{n}\right)-1$. By Proposition 3.1, we have

$$
\gamma_{r 3}^{\star}\left(C_{n}\right) \geq \omega(g)+1 \geq \gamma_{r 3}^{\star}\left(P_{n-1}\right)+1 \geq \frac{4(n-1)+1}{3}+1=\frac{4 n}{3} .
$$

Assume that $\left|f\left(v_{i}\right)\right|=0$ for each $i \in[n]$. If there exists some $k \in[n]$ such that $\left|f\left(v_{k} v_{k+1}\right)\right|=3$, then define $g: V\left(C_{n}-\left\{v_{k}, v_{k+1}\right\}\right) \cup E\left(C_{n}-\left\{v_{k}, v_{k+1}\right\}\right) \rightarrow \mathcal{P}([3])$ by $g\left(v_{k-1}\right)=f\left(v_{k-1}\right) \cup f\left(v_{k-1} v_{k}\right), g\left(v_{k+2}\right)=$ $f\left(v_{k+2}\right) \cup f\left(v_{k+1} v_{k+2}\right)$ and $g(x)=f(x)$ otherwise. Clearly, $g$ is a M3RDF of $P_{n-2}$ with weight at most $\gamma_{r 3}^{\star}\left(C_{n}\right)-3$. By Proposition 3.1, we have

$$
\gamma_{r 3}^{\star}\left(C_{n}\right) \geq \omega(g)+3 \geq \gamma_{r 3}^{\star}\left(P_{n-2}\right)+3 \geq \frac{4(n-2)-1}{3}+3=\frac{4 n}{3} .
$$

Now assume that $\left|f\left(v_{i} v_{i+1}\right)\right| \leq 2$ for each $i \in[n]$. Then the assignment of edges under $f$ should start one of the following: (i) $\{1\},\{2,3\},\{1\},\{2,3\} \ldots$, (ii) $\{2\},\{1,3\},\{2\},\{1,3\} \ldots$, (iii) $\{3\},\{1,2\},\{3\},\{1,2\} \ldots$. Thus, one can check that

$$
\gamma_{r 3}^{\star}\left(C_{n}\right) \geq\left\lceil\frac{n}{2}\right\rceil+2\left\lfloor\frac{n}{2}\right\rfloor \geq \frac{4 n}{3} .
$$

Therefore, we have $\gamma_{r 3}^{\star}\left(C_{n}\right) \geq \frac{4 n}{3}$.

Define $h: V\left(C_{n}\right) \cup E\left(C_{n}\right) \rightarrow \mathcal{P}([3])$ by $h\left(v_{1+3 i}\right)=\{1\}$ for $0 \leq i \leq \frac{n-3}{3}, h\left(v_{2+3 i} v_{3+3 i}\right)=[3]$ for $0 \leq i \leq \frac{n-3}{3}$ and $h(x)=\emptyset$ otherwise. It is easy to see that $h$ is a M3RDF of $C_{n}$ with weight $\frac{4 n}{3}$. Thus, we have $\gamma_{r 3}^{\star}\left(C_{n}\right)^{3}=$ $\frac{4 n}{3}$.

Proposition 3.3. For $n \geq 2$,

$$
\gamma_{r 3}^{\star}\left(K_{n}\right)= \begin{cases}\frac{3 n}{2} & \text { if } n \text { is even; } \\ \frac{3 n-1}{2} & \text { if } n \text { is odd. }\end{cases}
$$


Proof. Let $V\left(K_{n}\right)=\left\{v_{1}, \ldots, v_{n}\right\}$. If $n$ is odd, then define $g: V\left(K_{n}\right) \cup E\left(K_{n}\right) \rightarrow \mathcal{P}([3])$ by $g\left(v_{1}\right)=\{1\}$, $g\left(v_{2 i} v_{2 i+1}\right)=[3]$ for $1 \leq i \leq \frac{n-1}{2}$ and $g(x)=\emptyset$ otherwise. It is easy to see that $g$ is a M3RDF of $K_{n}$ with weight $\frac{3 n-1}{2}$. If $n$ is even, then define $h: V\left(K_{n}\right) \cup E\left(K_{n}\right) \rightarrow \mathcal{P}([3])$ by $h\left(v_{2 i-1} v_{2 i}\right)=[3]$ for $1 \leq i \leq \frac{n}{2}$ and $h(x)=\emptyset$ otherwise. It is easy to see that $h$ is a M3RDF of $K_{n}$ with weight $\frac{3 n}{2}$.

Now we claim that $\gamma_{r 3}^{\star}\left(K_{n}\right) \geq \frac{3 n}{2}$ if $n$ is even and $\gamma_{r 3}^{\star}\left(K_{n}\right) \geq \frac{3 n-1}{2}$ if $n$ is odd. One can easily check that $\gamma_{r 3}^{\star}\left(K_{2}\right)=3, \gamma_{r 3}^{\star}\left(K_{3}\right)=4$, and $\gamma_{r 3}^{\star}\left(K_{4}\right)=6$. We proceed by induction on $n$. Assume that $n \geq 5$.

Case 1. $n$ is odd.

Let $f$ be a $\gamma_{r 3}^{\star}\left(K_{n}\right)$-function. If $\sum_{i=1}^{n}\left|f\left(v_{i}\right)\right|=0$, then to dominate each $v_{i}$ we must have [3] $\subseteq \cup_{e \in N_{M}\left(v_{i}\right)} f(e)$ for each $v_{i} \in V\left(K_{n}\right)$. This implies that

$$
2 \gamma_{r 3}^{\star}\left(K_{n}\right)=\sum_{i=1}^{n} \sum_{e \in N_{M}\left(v_{i}\right)}|f(e)| \geq 3 n .
$$

Thus, $\gamma_{r 3}^{\star}\left(K_{n}\right)>\frac{3 n-1}{2}$.

Assume that $\sum_{i=1}^{n}\left|f\left(v_{i}\right)\right| \neq 0$. Without loss of generality, assume that $f\left(v_{1}\right) \neq \emptyset$. Define $g: V\left(K_{n}-v_{1}\right) \cup$ $E\left(K_{n}-v_{1}\right) \rightarrow \mathcal{P}([3])$ by $g\left(v_{i}\right)=f\left(v_{i}\right) \cup f\left(v_{1} v_{i}\right)$ for $2 \leq i \leq n$ and $g(x)=f(x)$ otherwise. Then $g$ is a M3RDF with weight at most $\gamma_{r 3}^{\star}\left(K_{n}\right)-1$. By the induction hypothesis, we have

$$
\gamma_{r 3}^{\star}\left(K_{n}\right) \geq \omega(g)+1 \geq \gamma_{r 3}^{\star}\left(K_{n-1}\right)+1=\frac{3(n-1)}{2}+1=\frac{3 n-1}{2} .
$$

Case 2. $n$ is even.

We choose a $\gamma_{r 3}^{\star}\left(K_{n}\right)$-function $f$ so that the size of $\left\{v_{i} \in V\left(K_{n}\right) \mid f\left(v_{i}\right) \neq \emptyset\right\}$ is as small as possible.

For an edge $v_{i} v_{j} \in E\left(K_{n}\right)$, if $\left|f\left(v_{i}\right)\right|+\left|f\left(v_{j}\right)\right|+\left|f\left(v_{i} v_{j}\right)\right|=3$, then define $h: V\left(K_{n}-\left\{v_{i}, v_{j}\right\}\right) \cup E\left(K_{n}-\right.$ $\left.\left\{v_{i}, v_{j}\right\}\right) \rightarrow \mathcal{P}([3])$ by $h\left(v_{k}\right)=f\left(v_{k}\right) \cup f\left(v_{k} v_{i}\right) \cup f\left(v_{k} v_{j}\right)$ for $v_{k} \in V\left(K_{n}\right) \backslash\left\{v_{i}, v_{j}\right\}$ and $h(x)=f(x)$ otherwise. Clearly $h$ is a M3RDF with weight $\gamma_{r 3}^{\star}\left(K_{n}\right)-3$. By the induction hypothesis, we have

$$
\gamma_{r 3}^{\star}\left(K_{n}\right) \geq \omega(h)+3 \geq \gamma_{r 3}^{\star}\left(K_{n-2}\right)+3=\frac{3(n-2)}{2}+3=\frac{3 n}{2} .
$$

Assume that $\left|f\left(v_{i}\right)\right|+\left|f\left(v_{j}\right)\right|+\left|f\left(v_{i} v_{j}\right)\right| \leq 2$ for any $v_{i}, v_{j} \in V\left(K_{n}\right)$.

For a vertex $v_{i} \in V\left(K_{n}\right)$, if $\left|f\left(v_{i}\right)\right|=2$, then define $g: V\left(K_{n}-v_{i}\right) \cup E\left(K_{n}-v_{i}\right) \rightarrow \mathcal{P}([3])$ by $g\left(v_{k}\right)=$ $f\left(v_{k}\right) \cup f\left(v_{k} v_{i}\right)$ for $v_{k} \in V\left(K_{n}\right) \backslash\left\{v_{i}\right\}$ and $g(x)=f(x)$ otherwise. Clearly $g$ is a M3RDF with weight $\gamma_{r 3}^{\star}\left(K_{n}\right)-2$. By the induction hypothesis, we have

$$
\gamma_{r 3}^{\star}\left(K_{n}\right) \geq \omega(g)+2 \geq \gamma_{r 3}^{\star}\left(K_{n-1}\right)+2=\frac{3(n-1)-1}{2}+2=\frac{3 n}{2} .
$$

Assume that $\left|f\left(v_{i}\right)\right| \leq 1$ for each $v_{i} \in V\left(K_{n}\right)$.

Let $N:=\left\{v_{i} \in V\left(\bar{K}_{n}\right) \mid f\left(v_{i}\right) \neq \emptyset\right\}$. For a fixed $v_{i} \in N$, suppose that there exists no $v_{j} \in V\left(K_{n}\right)$ such that $f\left(v_{i} v_{j}\right) \neq \emptyset$. Without loss of generality, we may assume that $f\left(v_{i}\right)=\{1\}$. To dominate elements in $\left\{v_{i} v_{k} \mid v_{k} \in V\left(K_{n}\right) \backslash\left\{v_{i}\right\}\right\}, \bigcup_{x \in N_{M}\left[v_{k}\right]} f(x)$ should contain 2 and 3 for each $v_{k} \in V\left(K_{n}\right) \backslash\left\{v_{i}\right\}$. Thus, we have $s_{2}, s_{3} \geq \frac{n}{2}$, where $s_{j}:=\left|\left\{x \in V\left(K_{n}-v_{i}\right) \cup E\left(K_{n}-v_{i}\right) \mid j \in f(x)\right\}\right|$. If $f(x) \neq \emptyset$ for all $x \in N_{M}\left[v_{k}\right] \backslash\left\{v_{i} v_{k}\right\}$, then $\sum_{x \in N_{M}\left[v_{k}\right]}|f(x)| \geq n$. This implies $\gamma_{r 3}^{\star}\left(K_{n}\right) \geq \frac{3 n}{2}$. Assume that for $v_{k} \in V\left(K_{n}\right) \backslash\left\{v_{i}\right\}$ there exists $x \in N_{M}\left[v_{k}\right] \backslash\left\{v_{i} v_{k}\right\}$ such that $f(x)=\emptyset$. Then to dominate such an element $x, \bigcup_{y \in N_{M}(x)} f(y)$ should contain 1. Thus, we have $s_{1} \geq \frac{n}{2}$ so that $\omega(f) \geq\left|f\left(v_{i}\right)\right|+s_{2}+s_{3}+s_{1}=1+\frac{3 n}{2}$.

Assume that for each $v_{i} \in N$ there exists a vertex $v_{j} \in V\left(K_{n}\right)$ such that $f\left(v_{i} v_{j}\right) \neq \emptyset$. Let $t$ be the size of set $N$. For $v_{j} \in V\left(K_{n}\right) \backslash N, \bigcup_{x \in N_{M}\left[v_{j}\right]} f(x)$ should contain [3]. For $v_{i} \in N, \bigcup_{x \in N_{M}\left(v_{i}\right)} f(x)$ contains at least one element. Thus, we have $\gamma_{r 3}^{\star}\left(K_{n}\right) \geq \frac{3(n-t)}{2}+\left(t+\frac{t}{2}\right)=\frac{3 n}{2}$. This completes the proof. 


\section{LOWER AND UPPER BOUNDS FOR TREES}

In this section, we provide lower and upper bounds for the middle 3-rainbow domination number of trees in terms of the matching number.

Theorem 4.1. For every tree $T$ of order $n, \gamma_{r 3}^{\star}(T) \leq n+\alpha^{\prime}(T)$.

Proof. Take a maximum matching $M$ in $T$. Let $U$ be the set of vertices which are not saturated by $M$. Define a function by $f(e)=[3]$ for $e \in M, f(u)=\{1\}$ for $u \in U$ and $f(x)=\emptyset$ otherwise. Clearly, $f$ is a M3RDF of $T$. Thus, $\gamma_{r 3}^{\star}(T) \leq|U|+3|M|=\left(n-2 \alpha^{\prime}(T)\right)+3 \alpha^{\prime}(T)=n+\alpha^{\prime}(T)$.

Lemma 4.2. Let $T$ be a tree and $P_{3}=u v w$ a path in $T$ with $\operatorname{deg}_{T}(v)=2$ and $\operatorname{deg}_{T}(w)=1$. Then $|f(u v)|+$ $|f(v)|+|f(v w)|+|f(w)| \geq 3$ for any $\gamma_{r 3}^{\star}(T)$-function $f$.

Proof. If $f(w)=\emptyset$, then to dominate $w$ it follows that $f(v w)=[3]$. If $f(v w)=\emptyset$, then to dominate $v w$ it follows that $f(u v) \cup f(v) \cup f(w)=[3]$. If $f(v)=\emptyset$, then to dominate $v$ it follows that $f(u v) \cup f(v w)=[3]$. In the above cases, we have $|f(u v)|+|f(v)|+|f(v w)|+|f(w)| \geq 3$. If $f(w), f(v w)$ and $f(v)$ are not empty, then $|f(u v)|+|f(v)|+|f(v w)|+|f(w)| \geq 3$. This completes the proof.

Theorem 4.3. For every tree $T, \gamma_{r 3}^{\star}(T) \geq \frac{5 \alpha^{\prime}(T)}{2}$.

Proof. We proceed by induction on the order $n$ of $T$. Obviously, the statement is true for all trees of order $n \leq 4$.

Let $T$ be a tree of order $n \geq 5$. Suppose that every tree $T^{\prime}$ of order $n^{\prime}(<n)$ satisfies $\gamma_{r 3}^{\star}\left(T^{\prime}\right) \geq \frac{5 \alpha^{\prime}\left(T^{\prime}\right)}{2}$. Let $M$ be a maximum matching in $T$. If $T$ is a star, then $\alpha^{\prime}(T)=1$ and so $n+1=\gamma_{r 3}^{\star}(T)>\frac{5}{2}$. Assume that $T$ is a double star $\operatorname{DS}_{p, q}$ with $p \geq q \geq 1$. Then $\alpha^{\prime}(T)=2$ and so $n+1=\gamma_{r 3}^{\star}(T)>5$. Now we assume that $T$ is neither a star or a double star. Then it is easy to see that $T$ has diameter at least four.

If $T$ has a pendant edge $u v$ such that $v$ is a leaf and $u v \notin M$, then for any $\gamma_{r 3}^{\star}(T)$-function $f$, the function $g: V(T-v) \cup E(T-v)$ defined by by $g(u)=f(u) \cup f(u v)$ and $g(x)=f(x)$ otherwise is a M3RDF of $T-v$ with weight at most $\omega(f)$. By the induction hypothesis, we have $\gamma_{r 3}^{\star}(T) \geq \gamma_{r 3}^{\star}(T-v) \geq \frac{5 \alpha^{\prime}(T-v)}{2}=\frac{5 \alpha^{\prime}(T)}{2}$. Thus, we assume the following.

Assumption 4.4. All pendant edges of $T$ belong to each maximum matching.

Then it follows that all support vertices have degree 2. If $\Delta(T)=2$, then $T$ is a path. So, the result follows by Proposition 3.1. From now on, assume that $\Delta(T) \geq 3$. Among all of diametrical paths in $T$, we choose $x_{0} x_{1}, \ldots, x_{d}$ so that it maximizes the size of $f\left(x_{d-2}\right)$. Root $T$ at $x_{0}$. It follows from Lemma 4.2 that $\left|f\left(x_{d-2} x_{d-1}\right)\right|+\left|f\left(x_{d-1}\right)\right|+\left|f\left(x_{d-1} x_{d}\right)\right|+\left|f\left(x_{d}\right)\right| \geq 3$. We divide our consideration into three cases.

Case 1. $\operatorname{deg}\left(x_{d-2}\right) \geq 3$.

First, suppose that there is a path $x_{d-2} y z$ in $T$ such that $z$ is a leaf and $y \notin\left\{x_{d-3}, x_{d-1}\right\}$. Then it follows from Lemma 4.2 that $|f(z)|+|f(y z)|+|f(y)|+\left|f\left(x_{d-2} y\right)\right| \geq 3$. Without loss of generality, we may assume that

$$
\left|f\left(x_{d-2} y\right)\right| \geq\left|f\left(x_{d-2} x_{d-1}\right)\right| .
$$

Let $T^{\prime}=T-\left\{x_{d-1}, x_{d}\right\}$. Then clearly $\alpha^{\prime}\left(T^{\prime}\right)=\alpha^{\prime}(T)-1$. Since both $f\left(x_{d-2} y\right)$ and $f\left(x_{d-2} x_{d-1}\right)$ are not [3], the assumption (4.1) implies that $\left.f\right|_{V\left(T^{\prime}\right) \cup E\left(T^{\prime}\right)}$ is a M3RDF of $T^{\prime}$ with weight at most $\omega(f)-3$. By the induction hypothesis, we have $\gamma_{r 3}^{\star}(T) \geq \gamma_{r 3}^{\star}\left(T^{\prime}\right)+3 \geq \frac{5 \alpha^{\prime}\left(T^{\prime}\right)}{2}+3=\frac{5\left(\alpha^{\prime}(T)-1\right)}{2}+3>\frac{5 \alpha^{\prime}(T)}{2}$.

Now assume that every element of $C\left(x_{d-2}\right) \backslash\left\{x_{d-1}\right\}$ is a leaf. Then it follows from Assumption 4.4 that $\left|C\left(x_{d-2}\right) \backslash\left\{x_{d-1}\right\}\right|=1$. Let $v \in C\left(x_{d-2}\right) \backslash\left\{x_{d-1}\right\}$. If $f(v)=\emptyset$, then $x_{d-2} v$ can not dominate $v$. Thus, we must have $f(v) \neq \emptyset$. We consider the following subcases. 
Subcase 1.1. $\left|f\left(x_{d-2} x_{d-1}\right)\right| \leq 1$.

This implies that $\left|f\left(x_{d-1}\right)\right|+\left|f\left(x_{d-1} x_{d}\right)\right|+\left|f\left(x_{d}\right)\right|=3$. Let $T^{\prime}=T-\left\{x_{d-1}, x_{d}\right\}$. Define $g: V\left(T^{\prime}\right) \cup$ $E\left(T^{\prime}\right) \rightarrow \mathcal{P}([3])$ by $g\left(x_{d-2}\right)=f\left(x_{d-2}\right) \cup f\left(x_{d-2} x_{d-1}\right)$ and $g(x)=f(x)$ otherwise. Clearly $g$ is a M3RDF of $T^{\prime}$ with weight $\omega(f)-3$. The result follows as above.

Subcase 1.2. $\left|f\left(x_{d-2} x_{d-1}\right)\right| \geq 2$.

It is easy to see that $\left|f\left(x_{d-1}\right)\right|+\left|f\left(x_{d-1} x_{d}\right)\right|+\left|f\left(x_{d}\right)\right|=4-\left|f\left(x_{d-2} x_{d-1}\right)\right|$. Let $T^{\prime}=T-\left\{x_{d-2}, v, x_{d-1}, x_{d}\right\}$. Define $g: V\left(T^{\prime}\right) \cup E\left(T^{\prime}\right) \rightarrow \mathcal{P}([3])$ by $g\left(x_{d-3}\right)=f\left(x_{d-3}\right) \cup f\left(x_{d-3} x_{d-2}\right)$ and $g(x)=f(x)$ otherwise. Clearly $g$ is a M3RDF of $T^{\prime}$ with weight $\omega(f)-5$. By the induction hypothesis, we have $\gamma_{r 3}^{\star}(T) \geq \gamma_{r 3}^{\star}\left(T^{\prime}\right)+5 \geq$ $\frac{5 \alpha^{\prime}\left(T^{\prime}\right)}{2}+5=\frac{5\left(\alpha^{\prime}(T)-2\right)}{2}+5=\frac{5 \alpha^{\prime}(T)}{2}$.

Case 2. $\operatorname{deg}\left(x_{d-2}\right)=2$ and $\operatorname{deg}\left(x_{d-3}\right) \geq 3$.

It follows from Assumption 4.4 that $x_{d-3} x_{d-2}, x_{d-1} x_{d} \in M$. If there exists a path $x_{d-3} x y z$ in $T$ such that $x \notin\left\{x_{d-2}, x_{d-4}\right\}$ and $z$ is a leaf. By Case 1 and Assumption 4.4, we may assume that $\operatorname{deg}(x)=\operatorname{deg}(y)=2$. It follows from Assumption 4.4 and $x_{d-3} x_{d-2} \in M$ that $x_{d-3} x, x y \notin M$. But, $(M \backslash\{y z\}) \cup\{x y\}$ is a maximum matching in $T$ not containing a pendant edge, a contradiction.

It follows from $\operatorname{deg}\left(x_{d-3}\right) \geq 3$ that there exists a path $x_{d-3} y z$ such that $\operatorname{deg}(y)=2$ and $z$ is a leaf. By Lemma 4.2, we have $\left|f\left(x_{d-2} x_{d-1}\right)\right|+\left|f\left(x_{d-1}\right)\right|+\left|f\left(x_{d-1} x_{d}\right)\right|+\left|f\left(x_{d}\right)\right| \geq 3$ and $\left|f\left(x_{d-3} y\right)\right|+|f(y)|+$ $|f(y z)|+|f(z)| \geq 3$.

Since $f$ is a $\gamma_{r 3}^{\star}(T)$-function, it is easy to see that

$$
4 \geq S\left(x_{d-3} y z\right) \geq 3 \text { and } 6 \geq S\left(x_{d-3} x_{d-2} x_{d-1} x_{d}\right) \geq 4,
$$

where $S\left(x_{d-3} y z\right):=\left|f\left(x_{d-3}\right)\right|+\left|f\left(x_{d-3} y\right)\right|+|f(y)|+|f(y z)|+|f(z)|$ and $S\left(x_{d-3} x_{d-2} x_{d-1} x_{d}\right):=\left|f\left(x_{d-3}\right)\right|+$ $\left|f\left(x_{d-3} x_{d-2}\right)\right|+\left|f\left(x_{d-2}\right)\right|+\left|f\left(x_{d-2} x_{d-1}\right)\right|+\left|f\left(x_{d-1}\right)\right|+\left|f\left(x_{d-1} x_{d}\right)\right|+\left|f\left(x_{d}\right)\right|$.

If $S\left(x_{d-3} x_{d-2} x_{d-1} x_{d}\right)=6$, then without loss of generality we may assume that $f\left(x_{d-3} x_{d-2}\right)=f\left(x_{d-1} x_{d}\right)=$ [3]. Let $T^{\prime}=T-\left\{x_{d-1}, x_{d}\right\}$. The function $\left.f\right|_{V\left(T^{\prime}\right) \cup E\left(T^{\prime}\right)}$ is a M3RDF of $T^{\prime}$ with weight $\omega(f)-3$. By the induction hypothesis, we have $\gamma_{r 3}^{\star}(T) \geq \gamma_{r 3}^{\star}\left(T^{\prime}\right)+3 \geq \frac{5 \alpha^{\prime}\left(T^{\prime}\right)}{2}+3=\frac{5\left(\alpha^{\prime}(T)-1\right)}{2}+3>\frac{5 \alpha^{\prime}(T)}{2}$.

If $S\left(x_{d-3} y z\right)=3$, then $|f(y)|+|f(y z)|+|f(z)|=3$ and $f\left(x_{d-3}\right)=f\left(x_{d-3} y\right)=\emptyset$. Let $T^{\prime}=T-\{y, z\}$. The function $\left.f\right|_{V\left(T^{\prime}\right) \cup E\left(T^{\prime}\right)}$ is a M3RDF of $T^{\prime}$ with weight $\omega(f)-3$. The result follows as above.

Now we assume that $S\left(x_{d-3} y z\right)=4$ and $5 \geq S\left(x_{d-3} x_{d-2} x_{d-1} x_{d}\right) \geq 4$. Then without loss of generality we may assume that $f\left(x_{d-3} y\right)=[3]$ and $f(z)=\{1\}$.

If $S\left(x_{d-3} x_{d-2} x_{d-1} x_{d}\right)=5$, then define $g: V(T) \cup E(T) \rightarrow \mathcal{P}([3])$ by $g\left(x_{d-2}\right)=\{1\}, g\left(x_{d-1} x_{d}\right)=[3]$ and $f\left(x_{d-3}\right)=f\left(x_{d-3} x_{d-2}\right)=f\left(x_{d-2} x_{d-1}\right)=f\left(x_{d-1}\right)=f\left(x_{d}\right)=\emptyset$. Clearly $g$ is a M3RDF of $T$ with weight $\omega(f)-1$, a contradiction.

Assume that $S\left(x_{d-3} x_{d-2} x_{d-1} x_{d}\right)=4$. Then without loss of generality we may assume that $f\left(x_{d-2}\right)=\{1\}$ and $f\left(x_{d-1} x_{d}\right)=[3]$. Let $T^{\prime}=T-\left\{x_{d-1}, x_{d}\right\}$. The function $\left.f\right|_{V\left(T^{\prime}\right) \cup E\left(T^{\prime}\right)}$ is a M3RDF of $T^{\prime}$ with weight $\omega(f)-3$. The result follows as above.

Case 3. $\operatorname{deg}\left(x_{d-2}\right)=2$ and $\operatorname{deg}\left(x_{d-3}\right)=2$.

If $f\left(x_{d-4} x_{d-3}\right)=[3]$, then it is easy to see that $\left|f\left(x_{d-3}\right)\right|+\left|f\left(x_{d-3} x_{d-2}\right)\right|+\left|f\left(x_{d-2}\right)\right|+\left|f\left(x_{d-2} x_{d-1}\right)\right|+$ $\left|f\left(x_{d-1}\right)\right|+\left|f\left(x_{d-1} x_{d}\right)\right|+\left|f\left(x_{d}\right)\right|=4$, since $f$ is a $\gamma_{r 3}^{\star}(T)$-function. Without loss of generality, we may assume that $f\left(x_{d-1} x_{d}\right)=[3], f\left(x_{d-2}\right)=\{1\}$ and $f\left(x_{d-3}\right)=f\left(x_{d-3} x_{d-2}\right)=f\left(x_{d-2} x_{d-1}\right)=f\left(x_{d-1}\right)=f\left(x_{d}\right)=\emptyset$. Let $T^{\prime}=T-\left\{x_{d-1}, x_{d}\right\}$. Then clearly $\alpha^{\prime}\left(T^{\prime}\right)=\alpha^{\prime}(T)-1$ and $\left.f\right|_{V\left(T^{\prime}\right) \cup E\left(T^{\prime}\right)}$ is a M3RDF of $T^{\prime}$ with weight at most $\omega(f)-3$. By the induction hypothesis, we have $\gamma_{r 3}^{\star}(T) \geq \gamma_{r 3}^{\star}\left(T^{\prime}\right)+3 \geq \frac{5 \alpha^{\prime}\left(T^{\prime}\right)}{2}+3=\frac{5\left(\alpha^{\prime}(T)-1\right)}{2}+3>$ $\frac{5 \alpha^{\prime}(T)}{2}$.

If ${ }^{2}\left(x_{d-4} x_{d-3}\right) \neq[3]$, then it is easy to see that $\left|f\left(x_{d-3}\right)\right|+\left|f\left(x_{d-3} x_{d-2}\right)\right|+\left|f\left(x_{d-2}\right)\right|+\left|f\left(x_{d-2} x_{d-1}\right)\right|+$ $\left|f\left(x_{d-1}\right)\right|+\left|f\left(x_{d-1} x_{d}\right)\right|+\left|f\left(x_{d}\right)\right|=5$, since $f$ is a $\gamma_{r 3}^{\star}(T)$-function. Without loss of generality, we may assume that $f\left(x_{d-3}\right)=f\left(x_{d}\right)=\{1\}, f\left(x_{d-2} x_{d-1}\right)=[3]$ and $f\left(x_{d-3} x_{d-2}\right)=f\left(x_{d-2}\right)=f\left(x_{d-1}\right)=f\left(x_{d-1} x_{d}\right)=\emptyset$. Let $T^{\prime}=T-T_{x_{d-3}}$. Define $g: V\left(T^{\prime}\right) \cup E\left(T^{\prime}\right) \rightarrow \mathcal{P}([3])$ by $g\left(x_{d-4}\right)=f\left(x_{d-4}\right) \cup f\left(x_{d-4} x_{d-3}\right)$ and $g(x)=f(x)$ otherwise. Then clearly $\alpha^{\prime}\left(T^{\prime}\right)=\alpha^{\prime}(T)-2$ and $g$ is a M3RDF of $T^{\prime}$ with weight at most $\omega(f)-5$. By the 
induction hypothesis, we have $\gamma_{r 3}^{\star}(T) \geq \gamma_{r 3}^{\star}\left(T^{\prime}\right)+5 \geq \frac{5 \alpha^{\prime}\left(T^{\prime}\right)}{2}+5=\frac{5\left(\alpha^{\prime}(T)-2\right)}{2}+5=\frac{5 \alpha^{\prime}(T)}{2}$. This completes the proof.

\section{The 3-RAinbow DOMATIC NUMBER FOR THE MidDle GRAPH OF PATHS AND CYCLES}

In this section, we determine the 3-rainbow domatic number for the middle graph of paths and cycles.

Theorem 5.1 (See [11]). If $G$ is a graph of order $n$, then $\gamma_{r k}(G) \cdot d_{r k}(G) \leq k n$.

Theorem 5.2 (See [11]). For every graph $G, d_{r k}(G) \leq \delta(G)+k$.

Proposition 5.3. For $n \geq 4$ and $n \equiv 0(\bmod 2), d_{r 3}\left(M\left(P_{n}\right)\right)=4$.

Proof. By Theorem 5.2, $d_{r 3}\left(M\left(P_{n}\right)\right) \leq 4$.

Let $P_{n}=v_{1} v_{2}, \ldots, v_{n}$. Define the 3 -rainbow dominating functions $f_{1}, f_{2}, f_{3}, f_{4}$ as follows:

$f_{1}\left(v_{1+2 i} v_{2+2 i}\right)=[3]$ for $0 \leq i \leq \frac{n-2}{2}$ and $f_{1}(x)=\emptyset$ otherwise,

$f_{2}\left(v_{1+2 i}\right)=\{1\}, f_{2}\left(v_{2+2 i}\right)=\{2\}$ for $0 \leq i \leq \frac{n-2}{2}, f_{2}\left(v_{2+2 i} v_{3+2 i}\right)=\{3\}$ for $0 \leq i \leq \frac{n-4}{2}$ and $f_{2}(x)=\emptyset$ otherwise,

$f_{3}\left(v_{1+2 i}\right)=\{2\}, f_{3}\left(v_{2+2 i}\right)=\{3\}$ for $0 \leq i \leq \frac{n-2}{2}, f_{3}\left(v_{2+2 i} v_{3+2 i}\right)=\{1\}$ for $0 \leq i \leq \frac{n-4}{2}$ and $f_{3}(x)=\emptyset$ otherwise,

$f_{4}\left(v_{1+2 i}\right)=\{3\}, f_{4}\left(v_{2+2 i}\right)=\{1\}$ for $0 \leq i \leq \frac{n-2}{2}, f_{4}\left(v_{2+2 i} v_{3+2 i}\right)=\{2\}$ for $0 \leq i \leq \frac{n-4}{2}$ and $f_{4}(x)=\emptyset$ otherwise.

Then clearly $f_{i}$ is a 3-rainbow dominating function on $M\left(P_{n}\right)$ for each $i$. Thus, $\left\{f_{1}, f_{2}, f_{3}, f_{4}\right\}$ is a family of 3-rainbow dominating functions on $M\left(P_{n}\right)$.

Proposition 5.4. For $n \geq 4, d_{r 3}\left(M\left(C_{n}\right)\right)=4$.

Proof. By Theorem 5.1, $\gamma_{r 3}\left(M\left(C_{n}\right)\right) \cdot d_{r 3}\left(M\left(C_{n}\right)\right) \leq 3 \cdot 2 n$. It follows from Proposition 3.2 that $d_{r 3}\left(M\left(C_{n}\right)\right) \leq 4$.

Let $C_{n}=v_{1} v_{2}, \ldots, v_{n} v_{1}$. We consider the following two cases.

Case 1. $n$ is even.

Extend the 3-rainbow dominating functions $f_{1}, f_{2}, f_{3}, f_{4}$ in Proposition 5.3 as follows:

$g_{1}\left(v_{n} v_{1}\right)=\emptyset$ and $g_{1}(x)=f_{1}(x)$ otherwise,

$g_{2}\left(v_{n} v_{1}\right)=\{1\}$ and $g_{2}(x)=f_{2}(x)$ otherwise,

$g_{3}\left(v_{n} v_{1}\right)=\{1\}$ and $g_{3}(x)=f_{3}(x)$ otherwise,

$g_{4}\left(v_{n} v_{1}\right)=\{1\}$ and $g_{4}(x)=f_{4}(x)$ otherwise.

Case 2. $n$ is odd.

Then $n-1 \geq 4$ is even. Let $f_{1}, f_{2}, f_{3}, f_{4}$ be the 3 -rainbow dominating functions on $M\left(P_{n-1}\right)$ given by Proposition 5.3. Extend them as follows:

$g_{1}\left(v_{n-1} v_{n}\right)=\emptyset, g_{1}\left(v_{n}\right)=\{1\}, g_{1}\left(v_{n} v_{1}\right)=\emptyset$ and $g_{1}(x)=f_{1}(x)$ otherwise,

$g_{2}\left(v_{n-1} v_{n}\right)=\{3\}, g_{2}\left(v_{n}\right)=\{2\}, g_{2}\left(v_{n} v_{1}\right)=\emptyset$ and $g_{2}(x)=f_{2}(x)$ otherwise,

$g_{3}\left(v_{n-1} v_{n}\right)=\{1\}, g_{3}\left(v_{n}\right)=\{3\}, g_{3}\left(v_{n} v_{1}\right)=\emptyset$ and $g_{3}(x)=f_{3}(x)$ otherwise,

$g_{4}\left(v_{n-1} v_{n}\right)=\emptyset, g_{4}\left(v_{n}\right)=\emptyset, g_{4}\left(v_{n} v_{1}\right)=[3]$ and $g_{4}(x)=f_{4}(x)$ otherwise.

In any case, clearly $g_{i}$ is a 3 -rainbow dominating function on $M\left(C_{n}\right)$ for each $i$. Thus, $\left\{g_{1}, g_{2}, g_{3}, g_{4}\right\}$ is a family of 3-rainbow dominating functions on $M\left(C_{n}\right)$.

Acknowledgements. The author would like to thank the anonymous reviewers for their comments. This research was supported by Basic Science Research Program through the National Research Foundation of Korea funded by the Ministry of Education (2020R1I1A1A01055403). 


\section{REFERENCES}

[1] J.A. Bondy and U.S.R. Murty, Graph Theory. Vol. 244 of Graduate Texts in Mathematics. Springer (2007).

[2] B. Brešar, Rainbow domination in graphs. In: Topics in Domination in Graphs, edited by T.W. Haynes, S.T. Hedetniemi and M.A. Henning. Springer International Publishing, Switzerland (2020).

[3] B. Brešar, M.A. Henning and D.F. Rall, Rainbow domination in graphs. Taiwanese J. Math. 12 (2008) $213-225$.

[4] G.J. Chang, J.J. Wu and X.D. Zhu, Rainbow domination on trees. Discrete Appl. Math. 158 (2010) 8-12.

[5] V.J.A. Cynthia and A. Kavitha, On 2 and 3-rainbow domination number of circulant graph $G(n ; \pm\{1,2,3\})$. Int. J. Math. Comput. Sci. 15 (2020) 661-670.

[6] M. Furuya, M. Koyanagi and M. Yokota, Upper bound on 3-rainbow domination in graphs with minimum degree 2. Discrete Optim. 29 (2018) 45-76.

[7] H. Gao, C. Xi and Y. Yang, The 3-rainbow domination number of the cartesian product of cycles. Mathematics 8 (2020) 65.

[8] T. Hamada and I. Yoshimura, Traversability and connectivity of the middle graph of a graph. Discrete Math. 14 (1976) $247-255$.

[9] K. Kim, Italian, 2-rainbow and Roman domination numbers in middle graphs, Submitted.

[10] Z.H. Shao, M.L. Liang, C. Yin, X.D. Xu, P. Pavlic and J. Zerovnik, On rainbow domination numbers of graphs. Inf. Sci. 254 (2014) 225-234.

[11] S.M. Sheikholeslami and L. Volkmann, The $k$-rainbow domatic number of a graph. Discuss. Math. Graph Theory 32 (2012) $129-140$.

[12] Y. Wang, X.L. Wu, N. Dehgardi, J. Amjadi, R. Khoeilar and J.B. Liu, $k$-rainbow domination number of $P_{3} \square P_{n} . M a t h e m a t i c s$ 7 (2019) 203

\section{Subscribe to Open (S2O) A fair and sustainable open access model}

This journal is currently published in open access under a Subscribe-to-Open model (S2O). S2O is a transformative model that aims to move subscription journals to open access. Open access is the free, immediate, online availability of research articles combined with the rights to use these articles fully in the digital environment. We are thankful to our subscribers and sponsors for making it possible to publish this journal in open access, free of charge for authors.

\section{Please help to maintain this journal in open access!}

Check that your library subscribes to the journal, or make a personal donation to the S2O programme, by contacting subscribers@edpsciences.org

More information, including a list of sponsors and a financial transparency report, available at: https://www. edpsciences.org/en/maths-s2o-programme 